\title{
Free vibration analysis of a DST-0285 truck crane considering changes in the system load configuration
}

\author{
Tomasz Geisler ${ }^{*}, 1$ \\ ${ }^{1}$ Institute of Mechanics and Machine Design Foundations, Faculty of Mechanical Engineering and \\ Computer Science, Czestochowa University of Technology, ul. J. H. Dąbrowskiego 73, 42-201, \\ Czestochowa, Poland
}

\begin{abstract}
A model of a truck crane, consisting of the fundamental units of the crane, is presented in this paper. The finite element method and a COSMOS/M package were applied to perform the modelling. Research into the influence of a change in the crane radius and load location on changes in the chosen free vibrations was carried out using the built models. Diagrams containing changes in the frequency of vibrations for chosen values of the crane radius and the load are presented and conclusions are drawn.
\end{abstract}

Keywords: truck crane, load, forms of vibrations, frequency

\section{Introduction}

The problem of modelling the dynamics of a truck crane support system subjected to a load is a complex one. To build an appropriate dynamic model of a crane, a knowledge of its basic work should be taken into account.

Crane work is associated with lifting and moving loads. This requires changes in the reciprocal locations of the fundamental systems of the crane. All the reciprocal positions of the crane units in the full range of their movements and the dynamic properties of all the units must be taken into account. This has been considered in many studies. A number of works have presented a multitude of problems related to descriptions of the influence of crane units on the dynamics of the crane system. A model that covers a full description of a load movement in relation to operational motions was presented in [1].

In the papers $[2,3]$ the influence of the support system, including the foundations, on the dynamics of a truck crane was analysed. The influence of changes in the mass of the load on the dynamics of the whole object was presented in [4]. The monograph [5] concerns modelling and research into the dynamics of a self-propelled truck crane.

In the paper [6] was analyzed of transverse damped vibrations in the system of changing the boom radius of a DST-0285 truck crane. Damped frequencies of vibrations and degree of amplitude decay of studied system were calculated.

\footnotetext{
* Corresponding author: geisler@imipkm.pcz.czest.pl

Reviewers: Marek Macko, Krzysztof Talaśka
} 
The construction of crane units and research into the dynamics of a chassis frame were presented in the papers $[7,8]$ which were sacrificed to the crane modelling. In the papers the dynamic stability of laboratory model of the truck crane was presented [9].

In the work [10] was formulated and solved the problem of transverse damped vibrations in a crane boom of a truck-mounted crane. The influence of structural damping on the support and slides of the boom and the change of the geometry of the system to the eigenvalues of the system are presented.

Later works considered the influence of the remaining units of a truck crane, including the crane boom, on the free vibrations of the crane. Works on the verification of a discretecontinuous model and FEM model of a truck crane were also published [11]. This work is a continuation of research into the crane system [12, 13, 14] taking into account changes in the crane radius and a variable value of the load. A paper on static analysis was published [15] in the frame of research into the crane support system.

The aim of this study was to build a complete truck crane model and to obtain modal shapes and the corresponding vibration frequency, which would illustrate the dynamic behaviour of the crane system for various configurations of its fundamental units while moving cargo.

Such a parametric model allows computations on the assumed reciprocal locations of the fundamental systems of the crane to be carried out.

Changes in the crane system configuration for the reciprocal locations of the fundamental systems of the crane comprehended in the usage documentation and found in crane exploitation are also possible. Due to number limitation of the created models, computations were performed for the selected operating configuration of the system.

This paper presents an analysis for three selected forms of vibrations. Those chosen forms gave the full picture of all crane systems linked as a whole. Changes in the frequency of vibrations for the chosen forms of the crane system in dependence on changes in: an elevation angle, the length of crane boom, load value and its location, were presented as the results of research.

\section{Computational model of a truck crane}

Modelling the crane support system requires basic units of the crane in their complete form, and the additional units, to be taken into consideration. The proposed model of the crane support system consists of coupled main crane units: the chassis frame, the rotating frame (body) with the embedded three-stage telescopic boom and the cordage. In the modelling the weight of the equipment and the units loading the system were taken into account.

Change in the configuration of the crane system is due to its operational movements. The movements are determined in a horizontal plane of rotation of the body frame with the telescopic boom in relation to the body frame and rotation of the boom regarding the body frame in a vertical lifting plane. The crane radius depends on an angle of inclination of telescopic crane boom and its variable length.

Load of the crane lifting system is carried out by the assumed load, suspended on ropes. Load model takes into account changes in the position of the load and associated elastic properties of cordage.

The location of the assumed load is determined by the combination of the aforementioned relative positions of the crane units and the length of the crane cordage. Computations were conducted on the selected positions of the body frame with the embedded telescopic boom in relation to the body frame.

The main units of a DST-0285 crane were designed and made in „Bumar-Fablok” Factory of Building Machinery and Locomotives in Chrzanow. The models are worked out on the basis of the submitted documentation. 
Construction of all main elements in the form of the main sheets, membranes, webs, ribs, beams and the other essential elements of construction was considered in the modelling crane units $[7,8,12]$.

The aim of the performed modelling was to obtain the crane model making research possible with the respect to determination of frequency and free vibrations of the crane system. The basic model was built with the use of the finite element method and a COSMOS/M package [16, 17].

In the modelling, the following basic structural elements were applied to linear analysis: shell3, solid, spring, rbar, mass.

A series of necessary simplifications, resulting from a large number of elements and associated with this complexity of construction, was applied in the model.

The basic model was built parametrically. This allows to change the relative positions of crane units and to modify all geometrical dimensions of the framework and the boom, for example the thickness of metal sheets. In the basic model, only the values of parameters, characteristics of system components and their mutual positions are being changed. The number of parameters was over 600 .

The load values as well as the length and the elasticity of ropes suspending the load are also introduced using the parametrical method.

The model of the analysed crane system consisting of connected chassis frame (1), body frame (2) and the telescopic crane boom (3), rope system (4) and the load (5) of a truck crane is presented in the figure (Fig. 1). Elasticity of the boom system (6) and elasticity of the support chassis system (7) were considered in the computations.

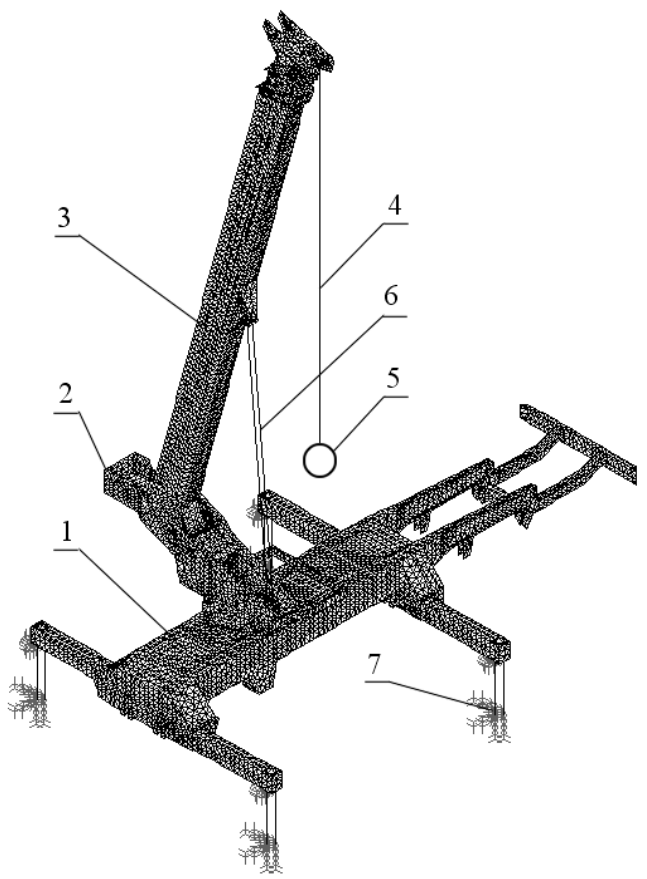

Fig. 1. The model of the truck crane system: (1) chassis frame, (2) body frame, (3) telescopic crane boom, (4) rope system, (5) load, (6) boom system, (7) support chassis system

The model was loaded with a cargo, placed as a weight suspended on a rope system. The load values of the model were given as the concentrated mass, suspended on elements modelling a rope system. 
Apart from the cargo load, system was loaded with the mass elements modelling majority of elements, construction units and the equipment of the crane. According to the documentation, the mass values of the crane equipment: the operator and driver cabins, main and auxiliary winches, counterweight, engine with a gearbox, front and rear suspension, wheels, lift and telescoping actuators were considered in modelling. The mass elements modelling crane units were placed in the areas of their actual location in the structure of the crane.

Suspension of the load on a spring rope system was taken into account and the equivalent elasticity value of the rope system was accepted for the computations [14] in the carried out modelling.

The computations were conducted assuming 6 strands of rope on the sheave block and accepting the rope length from the boom head to the main winch equalled to the boom length. Parameters of the rope were assumed as WS6x36 construction.

The equivalent elasticity value of the boom supporting actuator was considered. The elasticity adopted in the computations, varied along the length of the actuator and was associated with the value of the elevation angle of the telescopic boom.

The value of the boom support stiffness varied within $4-5 \times 10^{6}[\mathrm{~N} / \mathrm{m}]$. The model took into account the constant value of elasticity of the chassis support (support actuators) [7].

\section{Exemplary results of simulation research}

In the study of the dynamics of whole crane it is necessary to consider not only the behaviour of the telescopic boom, but also the other crane units. The dynamic behaviour of the linked crane units is substantial.

Consideration of the crane as a complex system should also include the movement of the telescopic boom mounted on the body frame, which then is mounted on the chassis frame.

The dynamic movement of the boom forces the movement of the whole body frame and a lifting element of the chassis frame.

Forced displacements of both frames are closely connected with the boom displacement, linking the movements of the major units into movement of the entire crane support system.

The study was conducted with the assumed configurations of the reciprocal positions of the crane units and lengths of rigging in order to obtain the influence of changes of boom outreach and load positions on free vibrations of the crane support system.

In order to perform the research, models of the body frame with a telescopic boom fixed to a body frame along the rotation plane were assumed, designation "+90" [13]. The models were built considering changes in the crane radius and load position.

Change in the crane radius encloses change in an angle of inclination and length of the boom while change in load of the crane encloses change in its value and length of its suspension. Considering constant location angle of the body frame with telescopic crane boom in relation to chassis frame, changeable parameters connected to changes in the crane radius were assumed:

- constant length of the boom $l t=10$ [m], changeable lifting angles of the boom $\alpha=0^{\circ}$, $25^{\circ}, 50^{\circ}, 75^{\circ}$.

- constant lifting angles of the boom $\alpha=0^{\circ}, 25^{\circ}, 50^{\circ}, 75^{\circ}$, changeable lengths of the boom $l t=10,15,20,24$ [m] (Fig. 2).

Changeable value of load location connected to the length of the crane cordage was given for all combinations of named parameters. The length of rope, $l=2,10,17,27$ [m], determined as the distance of cargo from the boom head at minimal permissible value $l=0.5[\mathrm{~m}]$, was assumed. 
Load $m$ was included from $m_{\min }=100[\mathrm{~kg}]$ to $m_{\max }=30000[\mathrm{~kg}]$. The values of the masses: $m=100,1000,5000,10000,20000,30000[\mathrm{~kg}]$ were accepted. Verifying computations for $l=0,5,12,22[\mathrm{~m}]$ and $m=0[\mathrm{~kg}]$ were conducted.

Changes in the crane radius and loads assumed in modelling exceeded the values determined by real hoisting capacity which were included in (DTR) documentation and the table of hoisting capacities. However, they were necessary to determine changes in the frequency of considered vibrations.

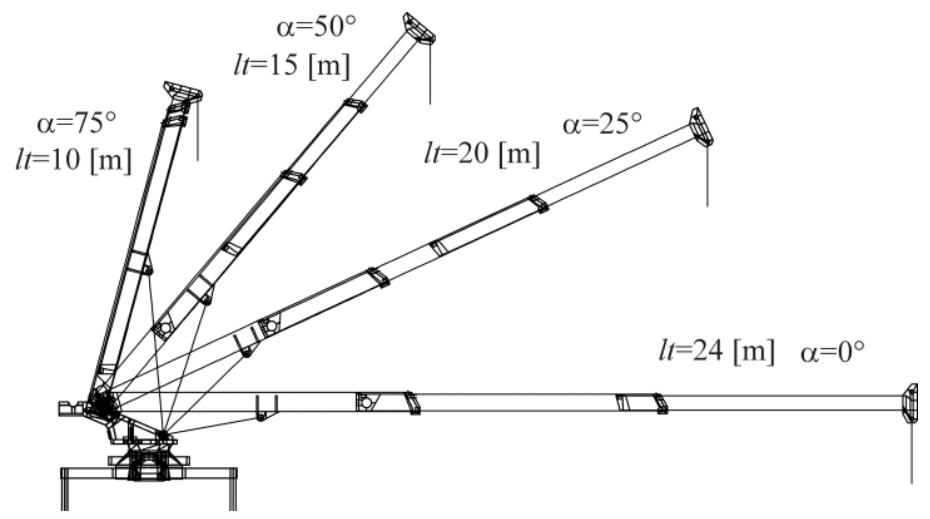

Fig. 2. Changes in the boom length and its location in relation to a body frame in lifting plane

Changes in the vibrational frequency of the chosen forms of free vibrations were presented as the results of solution to the problem of research into vibrations of the crane system. Changes in the values of the vibrational frequency for the three chosen forms of vibrations were put to the analysis. The choice of the analysed forms of vibrations resulted from the assumption that these forms have the highest influence on the dynamic behaviour of the crane support system.

For the chosen forms of vibrations the following denotations were assumed:

- form of flexural vibrations of the telescopic crane boom, denotation „G”, (frequency $\left.\omega_{1}\right)$,

- form of flexural vibrations of the boom together with frames, denotation "GR", (frequency $\omega_{2}$ ),

- form of flexural vibrations of the boom together with frames, denotation „GP”, (frequency $\omega_{3}$ ).

„G” form of vibrations contains mainly flexural vibrations of triple telescopic crane boom in the lifting plane (Fig. 3).

The next form, „GR”, contains flexural vibrations of the boom in interaction mainly with the body frame and at a lower rate with the chassis frame as the crane support system. For this form movement of the boom with the body frame produced rotation of the central part of the chassis frame around longitudinal axis (Fig. 4).

The form, denoted as „GP”, also contains flexural vibrations of the boom. In this case movement of the boom is connected to considerably higher displacements of whole support part of the chassis frame. The movements of the units take place in the vertical planes. For the "GP" form, displacement amplitude of the boom elements is comparable to displacements of the chassis frame elements (Fig. 5).

To determine vibrational frequency of the analysed forms, their identification was performed on the basis of an analysis of displacements of the crane units during animation of their movements. 
Figures (Figs. 3-5) present displacements of the boom and crane frames with the initial positions plotted for „G”, „GR” and „GP” forms.

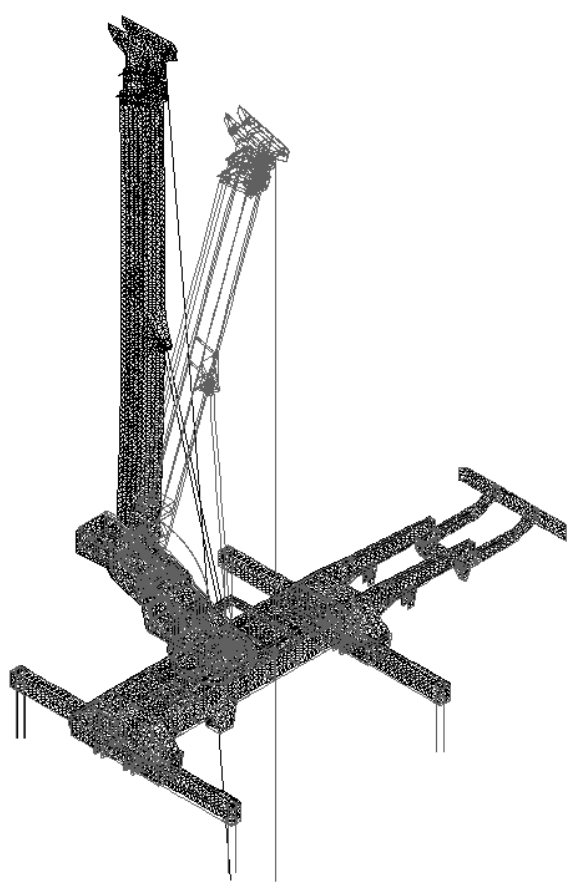

Fig. 3. Displacements of the boom for „G” form with the initial positions plotted

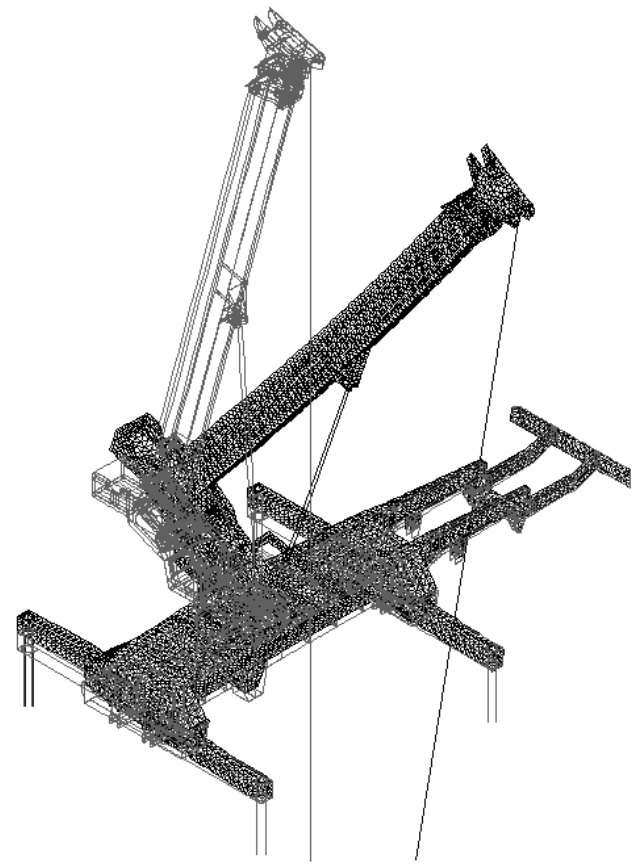

Fig. 4. Displacements of the boom and frames for „GR” form with the initial positions plotted 


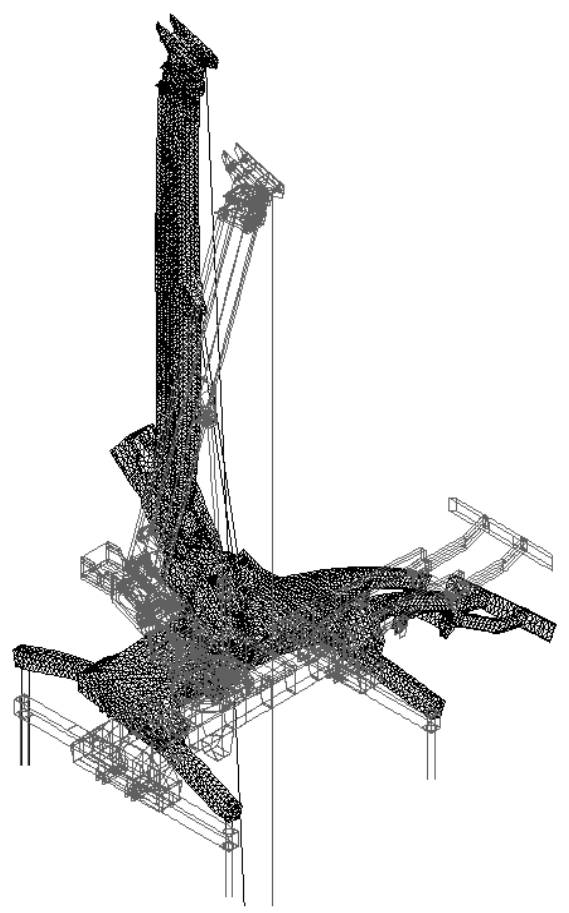

Fig. 5. Displacements of the boom and frames for „GP” form with the initial positions plotted

Computations were conducted on the chosen forms of vibrations containing the boom vibrations in the vertical lifting plane and for the displacements of body and chassis frames.

The obtained results are presented in diagrams containing courses of changes in the values of vibrational frequency in relation to chosen parameters.

Diagrams (Figs. 6-8) concern the influence of the boom $l t=10-24[\mathrm{~m}]$ and lifting angle $\left(\alpha=0^{\circ}, 75^{\circ}\right)$, for the constant value of the rope length $l=17[\mathrm{~m}]$ and changeable load $m=0-30000[\mathrm{~kg}]$.

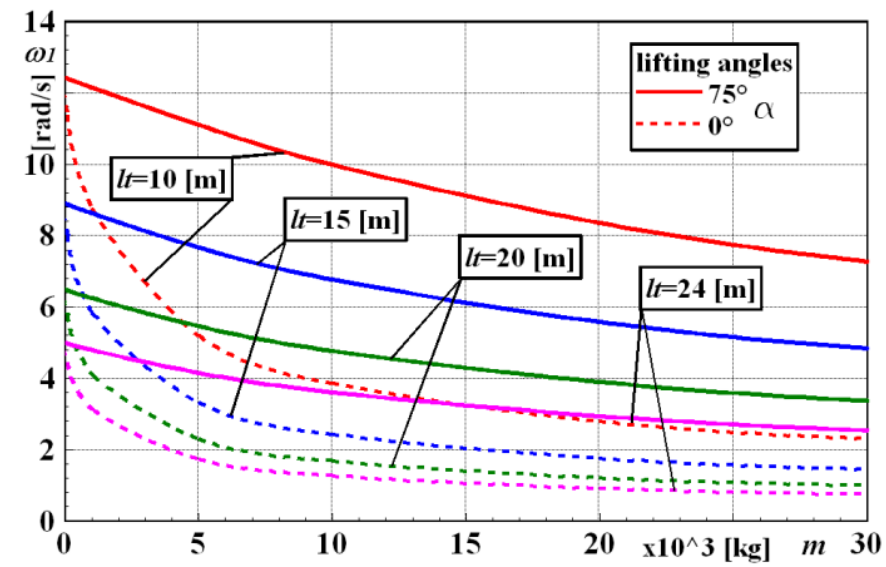

Fig. 6. Changes in vibrational frequency $\omega_{1}$ for „G” form 


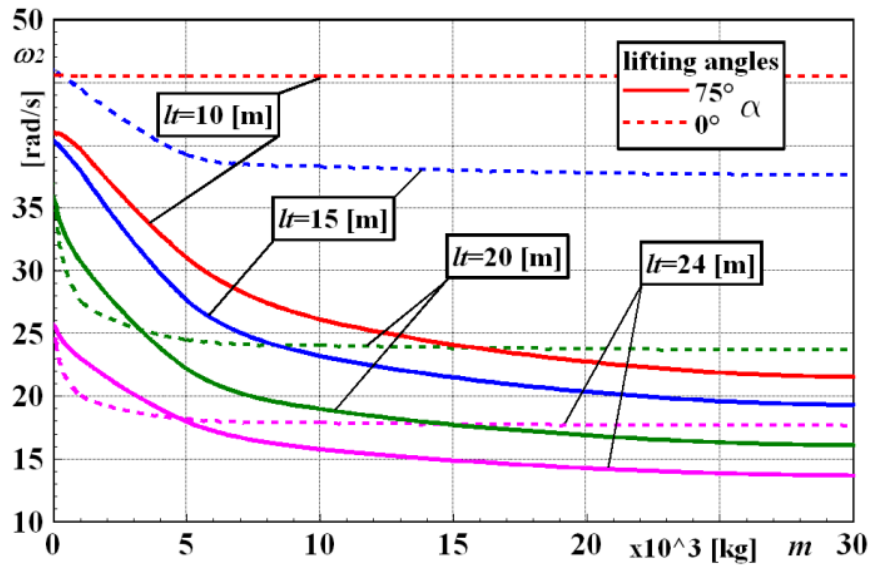

Fig. 7. Changes in vibrational frequency $\omega_{2}$ for „GR” form

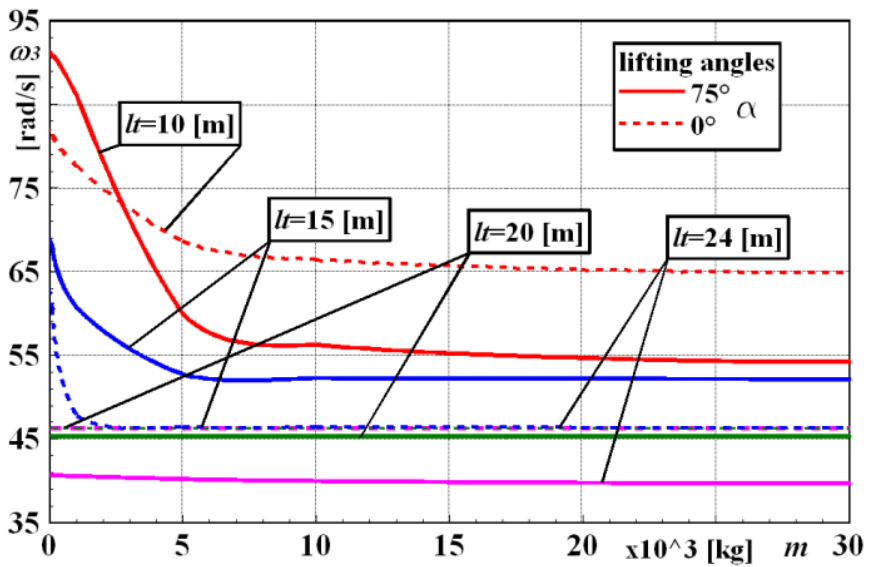

Fig. 8. Changes in vibrational frequency $\omega_{3}$ for „GP” form

In the diagrams plotted for two values of the boom length $(l t=10,24[\mathrm{~m}])$, one can observe decrease in vibrational frequencies $\omega_{1}, \omega_{2}$ and $\omega_{3}$ together with an increase in the value of the load $m$. At constant load together with decrease in an angle of inclination of the boom $\alpha$, the value of $\omega_{1}$ decreases for "G" form while for "GR" form the value of $\omega_{2}$ increases. This concerns every analysed length of telescopic crane boom.

For "GP" form together with decrease in the value of $\alpha$ angle one can observe significant change in value of $\omega_{3}$. For the greater lengths of the boom this influence is lesser. The course of line is very similar for the values: $l t=20$ and $24[\mathrm{~m}]$ and an angle $\alpha=0^{\circ}$.

Diagrams plotted in the "XYZ” coordinate system (3D) are placed in the further part of the paper. Vertical axis „Z” contains changes in vibrational frequency and horizontal axes $(\mathrm{X}, \mathrm{Y})$ contain changes in parameters $m$ and $l$, for the constant for example $l$ t.

Diagrams present data obtained for the higher number of computational models and contain surfaces built on the lines which represent changes in frequency of the tested forms in dependence on the chosen parameters. Changes in the shape of obtained surfaces allow for detailed description of analysed changes. 
Then chosen diagrams for the three analysed forms, for the constant length of the boom $l t=10[\mathrm{~m}]$, lifting angle $\alpha=0-75^{\circ}$ and rope length $l=17[\mathrm{~m}]$, considering changeable values of the load $m=0-30000[\mathrm{~kg}]$ were plotted (Figs. 9-11).

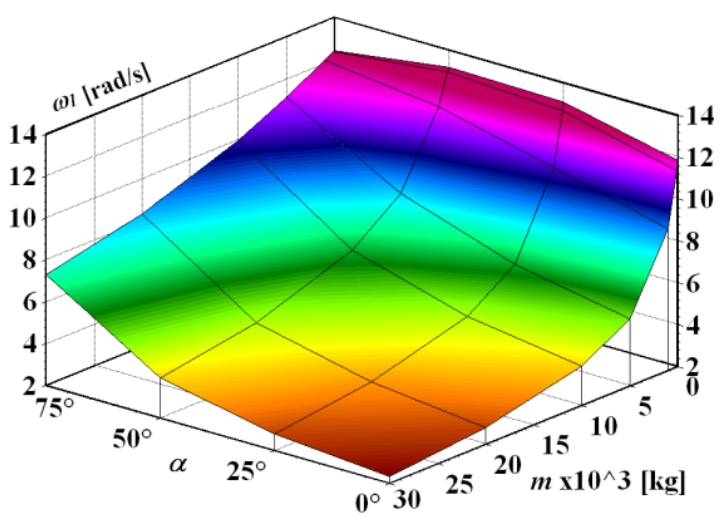

Fig. 9. Changes in vibrational frequency $\omega_{1}$, for „G” form

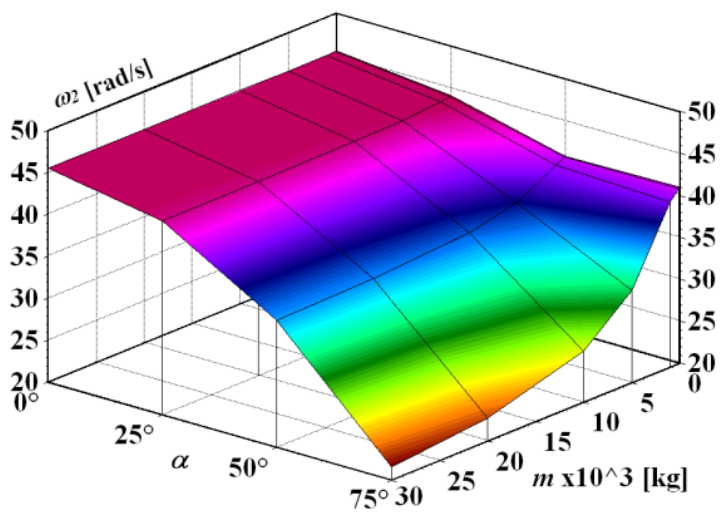

Fig. 10. Changes in vibrational frequency $\omega_{2}$, for „GR” form

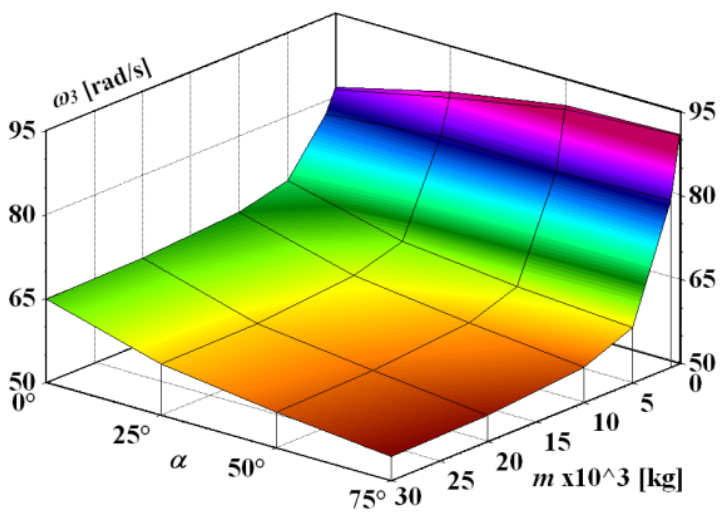

Fig. 11. Changes in vibrational frequency $\omega_{3}$, for „GP” form 
Increase in the value of the load $m$ leads to decrease in the value of frequencies of every tested forms of vibrations and depends on the lifting angle of the boom $\alpha$. For „G” and "GP" forms, increase in the value of the load leads to change in character of the curve dependent on the assumed value of the load.

Surfaces in the diagrams plotted for the other boom lengths have the similar course for "G” and „GR" forms. Range of changes and reduction of the value of frequency decrease together with increase in the boom length.

For "GP" form the shape of surface is changeable and depend on the assumed boom length. Range of changes in the value of frequency decreases together with an increase in the boom length and is the lowest for the boom length $l t=24$ [m]. Increase in the value of the load for constant length of rope and increase in the boom length leads to decrease in the values of vibrational frequency of the analysed forms. Character of decrease in the value of frequency is different and depends on the analysed lifting angle of the boom.

The next diagram (Fig. 12) was plotted for changes in frequencies of „GP” form. It contains the influence of rope length $l=2-27$ [m] on the changeable load $m=0-30000[\mathrm{~kg}]$ at the constant lifting angle $\alpha=75^{\circ}$.

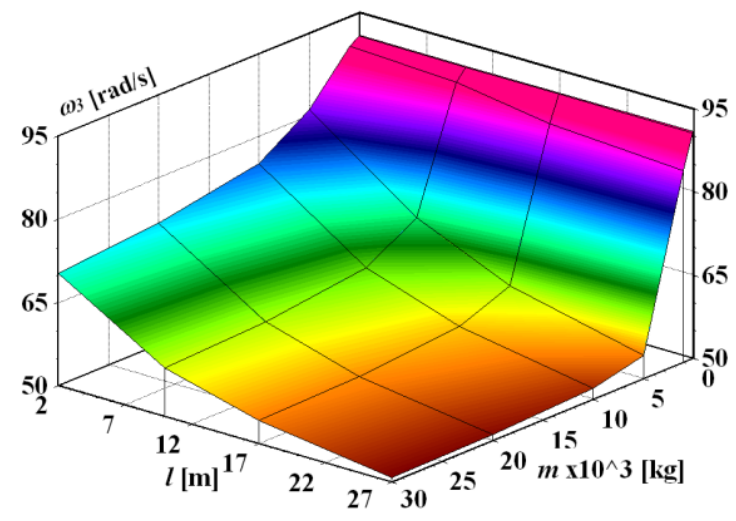

Fig. 12. Changes in vibrational frequency $\omega_{3}$, for „GP” form

The influence of the load location on the range of changes (decrease) in the value of frequency is connected to the length of rope system.

For the considered forms of frequencies, changes are most of all noticeable at the boom length $l t=10 \mathrm{~m}$.

For the highest assumed value of the load and rope lengths, decrease in the value of frequency equals for the forms: „G” $-8 \%$, ,GR” $-21.7 \%$, „GP” $-27.6 \%$, respectively. The range of changes decreases together with increase in the boom length and decrease in the angle of inclination.

An analysis of computations reveals the influence of change in the crane radius, which is a combination of lifting angle and the boom length and changes in rope length considering the changeable load, on changes in vibrational frequencies of the analysed forms.

\section{Conclusions}

The assumed aim of this work was realised through a series of computations on the worked out model to find the influence of different configurations of the crane support system on the dynamic behaviour of a truck crane during the load displacement. 
Consideration of the dynamic behaviour of the crane system took into account different forms of free vibrations containing movement of the telescopic crane boom as the main operating element and of the remaining units for their different reciprocal positions.

Chosen diagrams, which enable an evaluation of the influence of the assumed parameters on the behaviour of the crane system for the chosen forms of vibrations, were included in this paper. Consideration of changes in the crane radius in the lifting plane together with changes in the load revealed their essential influence on the dynamic behaviour of the crane support system.

The choice of three analysed forms of vibrations seemed to be justified due to their visible influence on the dynamic behaviour of the truck crane system.

Limiting the analysis of crane dynamics to only vibrations of the boom present for "G" form is insufficient. It is necessary to consider the consecutive forms of vibrations. The analysed forms of vibrations („GR”, „GP”) are complex forms of vibrations of crane units. They provide a wider picture of the dynamic behaviour of the crane units as a whole during changes in the crane radius and the load location.

In the research conducted into a comparison of MES model and the analytical model, change in curve for different lengths of ropes was stated for the „G” form. It depends on an increase in the load value pointed out in work [11].

Consideration of the chosen forms of vibrations and the consecutive forms presented in computations is essential for further analysis of the dynamics of a DST-0285 truck crane.

Research into the chosen forms containing changes in location of the telescopic crane boom with the body frame in relation to the chassis frame in the rotation plane is also justified. This is connected with the need to take into account the influence of the changeable rigidity of the lifting part of the chassis frame on the location of the remaining units of the crane.

The built models and conducted computations can be applied in experimental research into measurements of vibrational frequencies and into modal analysis of real system of a DST-0285 truck crane.

\section{References}

1. B. Posiadała, B. Skalmierski, L. Tomski, Motion of lifted load brought by kinematic forcing of the crane telescopic boom. Mechanism and Machine Theory 25 (5), 547-556 (1990)

2. B. Posiadała, Influence of crane support system on motion of the lifted load. Mechanism and Machine Theory 32 (1), 9-20 (1997)

3. Z. Towarek, The dynamic stability of a crane standing on soil during the rotation of the boom. International Journal of Mechanical Sciences 40 (6), 557-574 (1998)

4. J. Strzałko, J. Grabski, Dynamic analysis of a machine model with time-varying mass. Acta Mechanica 112, 173-186 (1995)

5. B. Posiadała (Ed.), Modelling, identification of models and research into dynamics of a truck mounted crane. (WNT, Warsaw: Scientific-Technical Publishing House, 2005) [in Polish]

6. W. Sochacki, M. Bold, Damped vibration of the system of changing the crane boom radius. Journal of Applied Mathematics and Computational Mechanics 14 (2), 111-122 (2015)

7. T. Geisler, B. Posiadała, Free vibration analysis of a chassis frame of a self-propelled truck crane. SYSTEMS - Journal of Transdisciplinary Systems Science, "Computer Aided Engineering" 7 (1), 199-205 (2002) [in Polish] 
8. T. Geisler, Research into the influence of a load on the vibrations of a chassis frame of a truck crane. Scientific Letters of Rzeszow University of Technology, Mechanics 60, 197-204 (2002) [in Polish]

9. W. Sochacki, The dynamic stability of a laboratory model of a truck crane. ThinWalled Structures 45, 927-930 (2007)

10. W. Sochacki, M. Bold, Effect of Structural Damping on Vibration of a Truck-Mounted Telescopic Crane. American Institute of Physics, AIP Conference Proceedings 1648, 850058-1- 850058-4 (2015)

11. T. Geisler, W. Sochacki, Modelling and research into the vibrations of a truck crane. Scientific research of the institute of mathematics and computer science 1 (10), 49-60 (2011)

12. T. Geisler, Research into the influence of a load on the vibrations of a crane boom of a DST-0285 truck crane. XXI National and First International Scientific Conference on Educational Theory of Machines and Mechanisms, Theory of Machines and Mechanisms, Bielsko-Biala - Szczyrk, 197-204 (2008) [in Polish]

13. T. Geisler, Research into the influence of a load location on the vibrations of a crane support system of a DST-0285 truck crane. Engineering Machines Problems 33, 15-24 (2009) [in Polish]

14. T. Geisler, Free vibration analysis of a crane support system of a DST-0285 truck crane considering changes in the system configuration, Acta mechanica et automatica 4 (1), 30-37 (2010) [in Polish]

15. T. Geisler, Static analysis of a crane support system of a DST-0285 truck crane. Mechanical Review (Przeglad Mechaniczny) 7/8, 42-48 (2012) [in Polish]

16. E. Rusiński, Finite Element Method. System COSMOS/M, WKi屯, Transport and Communication Publishers, Warsaw, (1994) [in Polish]

17. COSMOS/M User Guide Version 1.75. Structural Research and Analysis Corporation, Los Angeles, (1995) 